\title{
Methane Generation and Reductive Debromination of \\ Benzylic Position by Reconstituted Myoglobin Containing \\ Nickel Tetradehydrocorrin as a Model of Methyl-coenzyme M \\ Reductase
}

Supporting Information

Yuta Miyazaki, Koji Oohora* and Takashi Hayashi*

Department of Applied Chemistry, Graduate School of Engineering, Osaka University, Suita

565-0871, Japan 


\section{Table of Contents}

$\begin{array}{ll}\text { Instruments } & 3\end{array}$

$\begin{array}{lc}\text { Materials and methods } & 3\end{array}$

Synthesis of sodium 2-methylthioethanesufonate (methyl-coenzyme M) 3

Synthesis of 2-bromo-2-phenylpropane (cumyl bromide) 4

GC and GC/MS analyses for methane generation and reductive dehalogenation reaction 4

$\begin{array}{lr}\text { References } & 14\end{array}$ 


\section{Instruments}

Electrospray-ionization time-of-flight mass spectrometry (ESI-TOF MS) was carried out with a micrOTOF-II mass spectrometer (Bruker). UV-vis spectra were recorded with a BioSpec-nano spectrophotometer (Shimadzu) or a V-670 spectrophotometer equipped with an ETCS-761 Peltier thermostatted cell holder (JASCO). The $\mathrm{pH}$ measurements were made with an F-72 $\mathrm{pH}$ meter (Horiba). Air-sensitive manipulations were performed in a UNILab glove box (MBRAUN). Gas chromatography (GC) was conducted using a GC-2010 plus instrument (Shimadzu) equipped with a dielectric barrier discharge ionization detector (BID) and a SHINCARBON-ST 80-100 mesh micropacked column $(2.0 \mathrm{~m} \times 1.0 \mathrm{~mm}$ ID) using helium as a carrier gas. Gas chromatography/mass spectrometry (GC/MS) was performed using a GCMS-QP2010 Ultra gas chromatograph mass spectrometer (Shimadzu) equipped with a Rxi-5Sil MS 5\% diphenyl/95\% dimethyl polysiloxane capillary column $(30 \mathrm{~m} \times 0.25 \mathrm{~mm}$ ID $\times 0.25 \mu \mathrm{m} \mathrm{df})$ using helium as a carrier gas.

\section{Materials and methods.}

All chemicals were purchased from Sigma-Aldrich, TCI, Wako, and Nacalai and were used as received unless otherwise noted. Authentic methane gas $(0.399 \%(v / v)$, balancer: Ar) was purchased from GL Sciences Inc. Deuterium oxide (deuteration degree min. 99.9\%) was purchased from Merck KGaA. Ultrapure water was obtained using a Millipore Integral 3 apparatus. Nickel tetradehydrocorrin $(\mathrm{Ni}(\mathrm{TDHC}))^{\mathrm{S} 1}$ and cobalt tetradehydrocorrin $(\mathrm{Co}(\mathrm{TDHC}))^{\mathrm{S} 2}$ were synthesized according to the procedures outlined in our previous reports. ApoMb, ${ }^{\mathrm{S} 3} \mathrm{rMb}\left(\mathrm{Ni}\right.$ (TDHC)), ${ }^{\mathrm{S} 1}$ and $\mathrm{rMb}\left(\mathrm{Co}^{\mathrm{II}}(\mathrm{TDHC})\right)^{\mathrm{S} 2}$ were prepared using previously published methods. Methyl-coenzyme $\mathrm{M}^{\mathrm{S} 4}$ and cumyl bromide ${ }^{\mathrm{S} 5}$ were synthesized according to the reported procedures.

\section{Synthesis of sodium 2-methylthioethanesufonate (methyl-coenzyme M)}

To a solution of sodium 2-mercaptoethane sulfonate (coenzyme M sodium salt) (9.12 g, $55.5 \mathrm{mmol}$ ) in methanol $(100 \mathrm{~mL})$ was added $28 \%$ sodium methoxide in methanol $(10.9 \mathrm{~mL}, 55.0 \mathrm{mmol}, 0.99$ eq.) and methyl iodide (3.5 mL, $56.1 \mathrm{mmol}, 1.01$ eq.) under an $\mathrm{N}_{2}$ atmosphere. The mixture was stirred overnight at $60{ }^{\circ} \mathrm{C}$. After evaporation under reduced pressure to remove solvent, the crude product was reprecipitated from acetone/water to yield sodium 2-methylthioethanesufonate as white powder $(9.87 \mathrm{~g}, 55.4 \mathrm{mmmol}, 99 \%$ yield$)$, whose spectra match literature report. ${ }^{\mathrm{S} 4}$

${ }^{1} \mathrm{H}$ NMR (400 MHz, $\mathrm{D}_{2} \mathrm{O}, 1,4$-dioxane $\left.=3.75\right): \delta 3.17(\mathrm{t}, J=8.0 \mathrm{~Hz}, 2 \mathrm{H}), 2.85(\mathrm{t}, J=8.0 \mathrm{~Hz}, 2 \mathrm{H})$, $2.15(\mathrm{~s}, 3 \mathrm{H})$.

${ }^{13} \mathrm{C}$ NMR (100 MHz, $\mathrm{D}_{2} \mathrm{O}, 1$,4-dioxane $\left.=67.19\right): \delta 51.23,28.25,14.87$.

HRMS (ESI, positive mode, $m / z$ ): calcd. for $\mathrm{C}_{3} \mathrm{H}_{8} \mathrm{O}_{3} \mathrm{~S}_{2}[\mathrm{M}+\mathrm{H}]^{+}$154.983; found 154.983 . 


\section{Synthesis of 2-bromo-2-phenylpropane (cumyl bromide)}

A mixture of 2-phenyl-2-propanol $(1.07 \mathrm{~g}, 7.86 \mathrm{mmol})$ and bromotrimethylsilane $(1.53 \mathrm{~mL}, 11.8$ mmol, 1.5 eq.) was stirred overnight at room temperature under an $\mathrm{N}_{2}$ atmosphere. Evaporation under reduced pressure $(0.75 \mathrm{mmHg})$ at $40{ }^{\circ} \mathrm{C}$ removed volatile product and provided 2-bromo-2phenylpropane as a colorless oil (1.45 g, $7.26 \mathrm{mmol}, 92 \%$ yield), whose spectra match literature report. ${ }^{\mathrm{S} 5}$

${ }^{1} \mathrm{H}$ NMR (400 MHz, $\left.\mathrm{CDCl}_{3}\right): \delta 7.62(\mathrm{~m}, 2 \mathrm{H}), 7.34(\mathrm{~m}, 2 \mathrm{H}), 7.28(\mathrm{~m}, 1 \mathrm{H}), 2.20(\mathrm{~s}, 3 \mathrm{H})$.

${ }^{13} \mathrm{C} \mathrm{NMR}\left(100 \mathrm{MHz}, \mathrm{CDCl}_{3}\right): \delta 146.89,128.40,127.87,125.85,64.23,35.60$.

HRMS (ESI, positive mode, $m / z$ ): calcd. for $\mathrm{C}_{9} \mathrm{H}_{12} \mathrm{Br}[\mathrm{M}+\mathrm{H}]^{+}$199.012; found 199.015.

\section{GC and GC/MS analyses for methane generation and reductive dehalogenation reaction}

Analyses for methane generartion from methyl donors and reductive dehalogenation of benzyl bromide derivatives were carried out along with the following conditions.

Measurement condition of GC for methane generation:

split ratio $5: 1$, injector temperature $=200{ }^{\circ} \mathrm{C}$, detector temperature $=250{ }^{\circ} \mathrm{C}$, column temperature $=$ $60{ }^{\circ} \mathrm{C}(6 \mathrm{~min}$ hold $)-40{ }^{\circ} \mathrm{C} / \mathrm{min}-200{ }^{\circ} \mathrm{C}(5.5 \mathrm{~min}$ hold $)$, flow rate $55.8 \mathrm{~mL} / \mathrm{min}$.

Measurement condition of GC/MS for reductive dehalogenation reactions with benzyl bromide, 1phenylthyl bromide, cumyl bromide, and para-substituted benzyl bromides $\left(-\mathrm{CH}_{3},-\mathrm{Cl}\right.$, and $\left.-\mathrm{CF}_{3}\right)$ : split less, injector temperature $=250^{\circ} \mathrm{C}$, interface temperature $=250{ }^{\circ} \mathrm{C}$, column temperature $=40^{\circ} \mathrm{C}$ (3 min hold) $-25^{\circ} \mathrm{C} / \mathrm{min}$ until $180^{\circ} \mathrm{C}-50^{\circ} \mathrm{C} / \mathrm{min}$ until $250^{\circ} \mathrm{C}(5 \mathrm{~min}$ hold $)$, flow rate $50 \mathrm{~mL} / \mathrm{min}$.

Measurement condition of GC/MS for reductive dehalogenation reactions with para-substituted benzyl bromids $\left(-\mathrm{OCH}_{3},-\mathrm{I},-\mathrm{Br},-\mathrm{CN}\right.$, and $\left.-\mathrm{NO}_{2}\right)$ :

split less, injector temperature $=250{ }^{\circ} \mathrm{C}$, interface temperature $=250{ }^{\circ} \mathrm{C}$, column temperature $=$ $100{ }^{\circ} \mathrm{C}(3 \mathrm{~min}$ hold $)-12.5^{\circ} \mathrm{C} / \mathrm{min}$ until $200^{\circ} \mathrm{C}-50{ }^{\circ} \mathrm{C} / \mathrm{min}$ until $250^{\circ} \mathrm{C}$ (5 min hold), flow rate 50 $\mathrm{mL} / \mathrm{min}$.

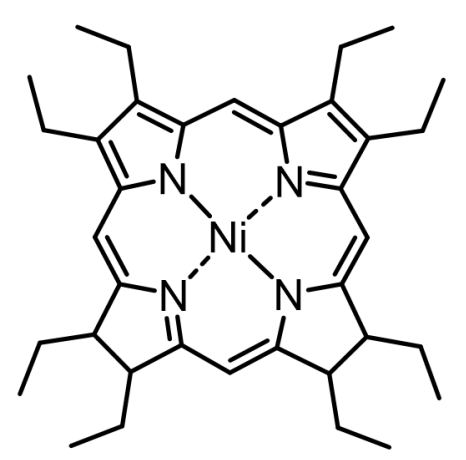

Figure S1. Chemical structure of $\mathrm{Ni}(\mathrm{OEiBC})$. 
Table S1. Products of the reaction of $\mathrm{Ni}^{\mathrm{I}}(\mathrm{TDHC})$ with methyl donors. ${ }^{\mathrm{a}, \mathrm{b}}$

\begin{tabular}{|c|c|c|c|c|}
\hline & $\begin{array}{c}\mathrm{CH}_{3}-\mathrm{R} \\
=-\mathrm{OTs},-\mathrm{S}^{+}\left(\mathrm{CH}_{3}\right)_{2}\end{array}$ & $\begin{array}{c}\underset{\mathrm{Nil}(\mathrm{TDHC})}{\text { dithionite }} \\
\underset{\text { buffer }(\mathrm{pH} 7.0)}{25^{\circ} \mathrm{C}}\end{array}$ & $\mathrm{CH}_{4}$ & \\
\hline substrate & methane (nmol) & $\begin{array}{l}\text { TON for } \\
\text { methane }\end{array}$ & ethane (nmol) & $\begin{array}{l}\text { TON for } \\
\text { ethane }\end{array}$ \\
\hline $\begin{array}{l}\text { methyl } p \text { - } \\
\text { toluenesulfonate }\end{array}$ & n.d. & - & n.d. & - \\
\hline $\begin{array}{l}\text { trimethylsulfonium } \\
\text { iodide }\end{array}$ & n.d. & - & n.d. & - \\
\hline
\end{tabular}

a General reaction conditions: $\left[\mathrm{Ni}^{\mathrm{I}}(\mathrm{TDHC})\right]=45 \mu \mathrm{M}$, [dithionite] $=1.0 \mathrm{mM}$, [methyl donors] $=5.0$ $\mathrm{mM}$ in potassium phosphate buffer $(100 \mathrm{mM}, \mathrm{pH} 7.0)$ at $25^{\circ} \mathrm{C}$ for $7 \mathrm{~h}$ under an $\mathrm{N}_{2}$ atmosphere. Products were identified and quantified by GC.

${ }^{\mathrm{b}}$ n.d.: not detected.

Table S2. Products of the reaction of dithionite with methyl donors. ${ }^{\mathrm{a}, \mathrm{b}}$

\begin{tabular}{|c|c|c|c|c|}
\hline & $\mathrm{CH}_{3}-\mathrm{R}$ & $\begin{array}{c}\underset{\text { buffer }(\mathrm{pH} 7.0)}{25^{\circ} \mathrm{C}} \\
\underset{1}{\text { dithionite }}\end{array}$ & $\mathrm{CH}_{4}$ & \\
\hline \multicolumn{5}{|c|}{$\mathbf{R}=-\mathrm{OTs},-\mathrm{S}^{+}\left(\mathrm{CH}_{3}\right)_{2}$} \\
\hline substrate & methane (nmol) & $\begin{array}{l}\text { TON for } \\
\text { methane }\end{array}$ & ethane (nmol) & $\begin{array}{c}\text { TON for } \\
\text { ethane }\end{array}$ \\
\hline $\begin{array}{l}\text { methyl } p \text { - } \\
\text { toluenesulfonate }\end{array}$ & n.d. & - & n.d. & - \\
\hline $\begin{array}{l}\text { trimethylsulfonium } \\
\text { iodide }\end{array}$ & n.d. & - & n.d. & - \\
\hline
\end{tabular}

a General reaction conditions: [dithionite] $=1.0 \mathrm{mM}$, [methyl donors] $=5.0 \mathrm{mM}$ in potassium phosphate buffer ( $100 \mathrm{mM}, \mathrm{pH} 7.0)$ at $25^{\circ} \mathrm{C}$ for $7 \mathrm{~h}$ under an $\mathrm{N}_{2}$ atmosphere. Products were identified and quantified by GC.

${ }^{\mathrm{b}}$ n.d.: not detected. 
(a)

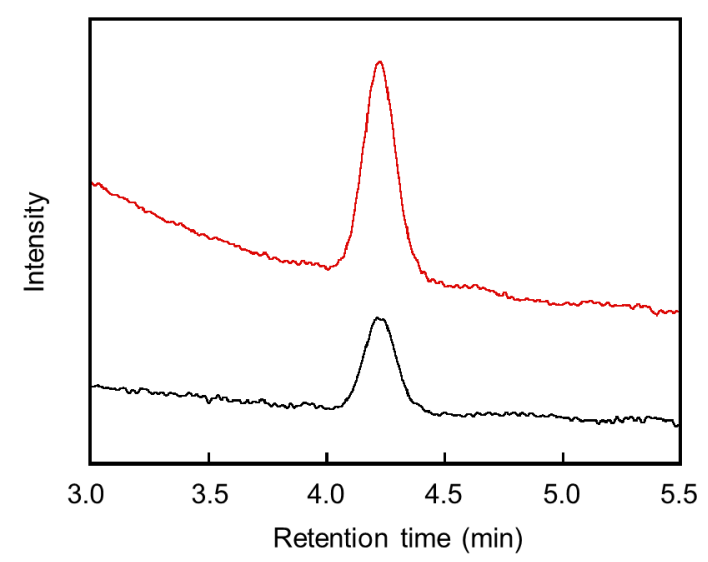

(b)

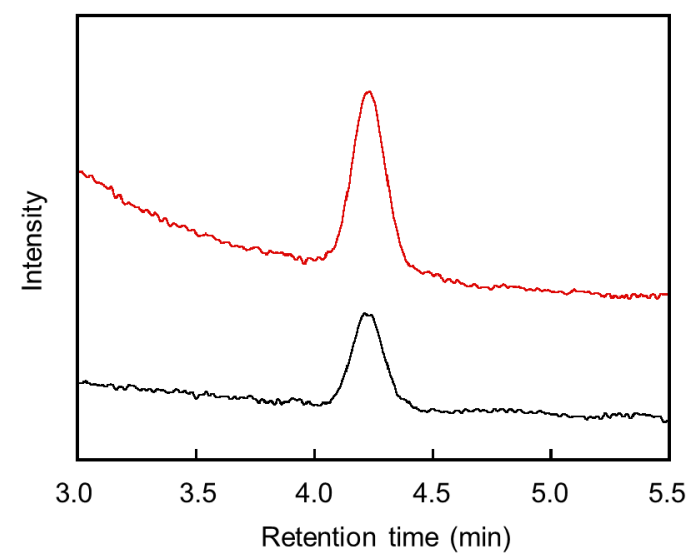

Figure S2. GC traces of the reaction of $\mathrm{rMb}\left(\mathrm{Ni}{ }^{\mathrm{I}}(\mathrm{TDHC})\right.$ ) with (a) methyl $p$-toluenesulfonate (red color) or (b) trimethylsulfonium iodide (red color) in the presence of dithionite. Authentic methane gas are shown with black lines.

Table S3. Products of the reaction of $\mathrm{rMb}\left(\mathrm{Ni}^{\mathrm{I}}(\mathrm{TDHC})\right.$ ) with benzyl bromide under different $\mathrm{pH}$ values. $^{\mathrm{a}, \mathrm{b}}$

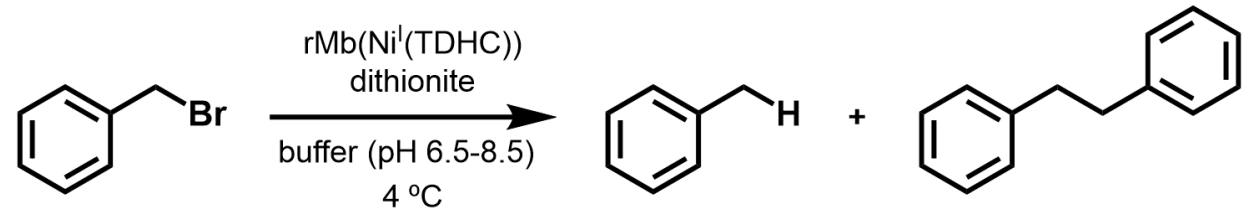

\begin{tabular}{ccccc}
\hline $\mathrm{pH}$ & {$[$ toluene] $(\mu \mathrm{M})$} & TON for toluene & $\begin{array}{c}{[\text { dibenzyl }]} \\
(\mu \mathrm{M})\end{array}$ & $\begin{array}{c}\text { TON for } \\
\text { dibenzyl }\end{array}$ \\
\hline 6.5 & $8.5 \pm 0.85$ & $0.19 \pm 0.019$ & n.d. & - \\
7.0 & $9.8 \pm 0.31$ & $0.22 \pm 0.007$ & n.d. & - \\
7.5 & $5.0 \pm 0.14$ & $0.11 \pm 0.003$ & n.d. & - \\
8.0 & $0.9 \pm 0.04$ & $0.02 \pm 0.001$ & n.d. & - \\
8.5 & $0.5 \pm 0.19$ & $0.01 \pm 0.004$ & n.d. & - \\
\hline
\end{tabular}

${ }^{\mathrm{a}}$ General reaction conditions: $\left[\mathrm{rMb}\left(\mathrm{Ni}^{\mathrm{I}}(\mathrm{TDHC})\right)\right]=45 \mu \mathrm{M}$, [dithionite] $=1.0 \mathrm{mM}$, [benzyl bromide] $=1.0 \mathrm{mM}$ in potassium phosphate buffer $(100 \mathrm{mM})$ containing $1 \%(v / v)$ acetonitrile at $4{ }^{\circ} \mathrm{C}$ for $2 \mathrm{~h}$ under an $\mathrm{N}_{2}$ atmosphere. Products were identified and quantified by GC/MS.

${ }^{b}$ n.d.: not detected. 

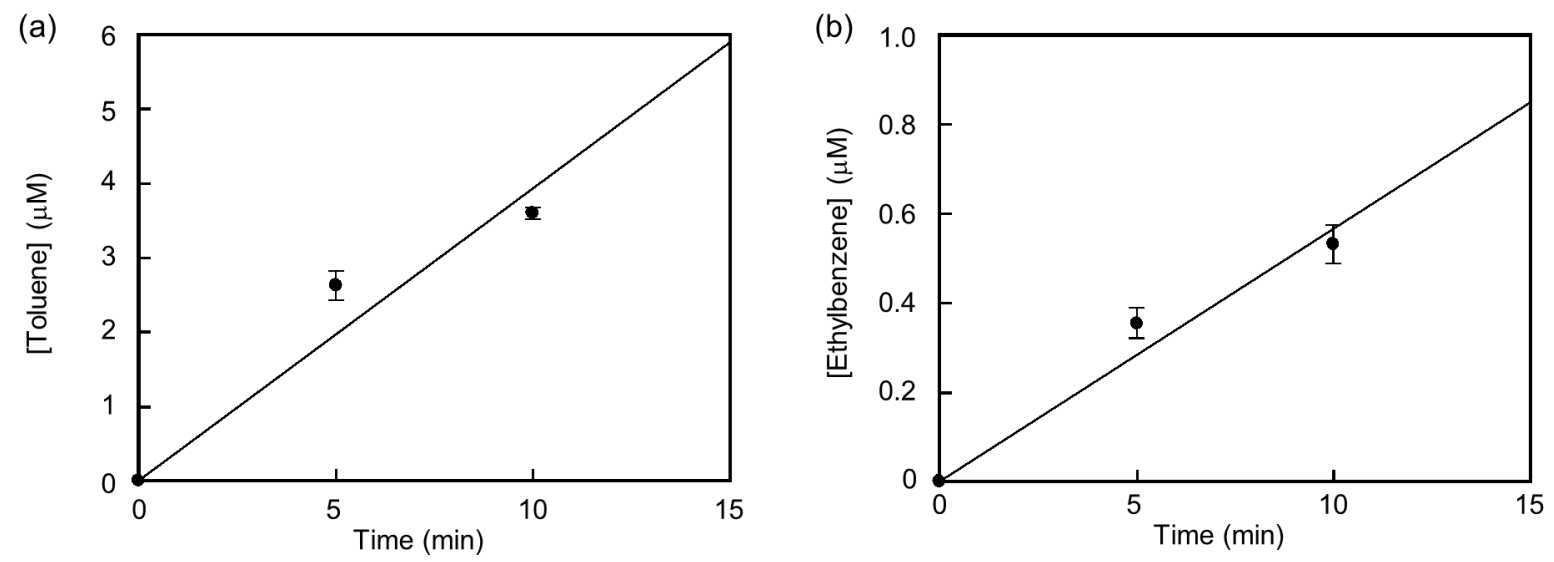

Figure S3. Time-course plots of toluene derivatives generation by the reductive dehalogenation of (a) benzyl bromide and (b) 1-phenylethyl bromide using $\mathrm{rMb}\left(\mathrm{Ni}^{\mathrm{I}}(\mathrm{TDHC})\right)$. Conditions: $\left[\mathrm{rMb}\left(\mathrm{Ni}^{\mathrm{I}}(\mathrm{TDHC})\right)\right]=45 \mu \mathrm{M}$, [dithionite] $=1.0 \mathrm{mM}$, [benzyl bromide] or [1-phenylethyl bromide] $=$ $1.0 \mathrm{mM}$ in potassium phosphate buffer $(100 \mathrm{mM}, \mathrm{pH} 7.0)$ containing $1 \%(v / v)$ acetonitrile at $4{ }^{\circ} \mathrm{C}$ under an $\mathrm{N}_{2}$ atmosphere.

(a)

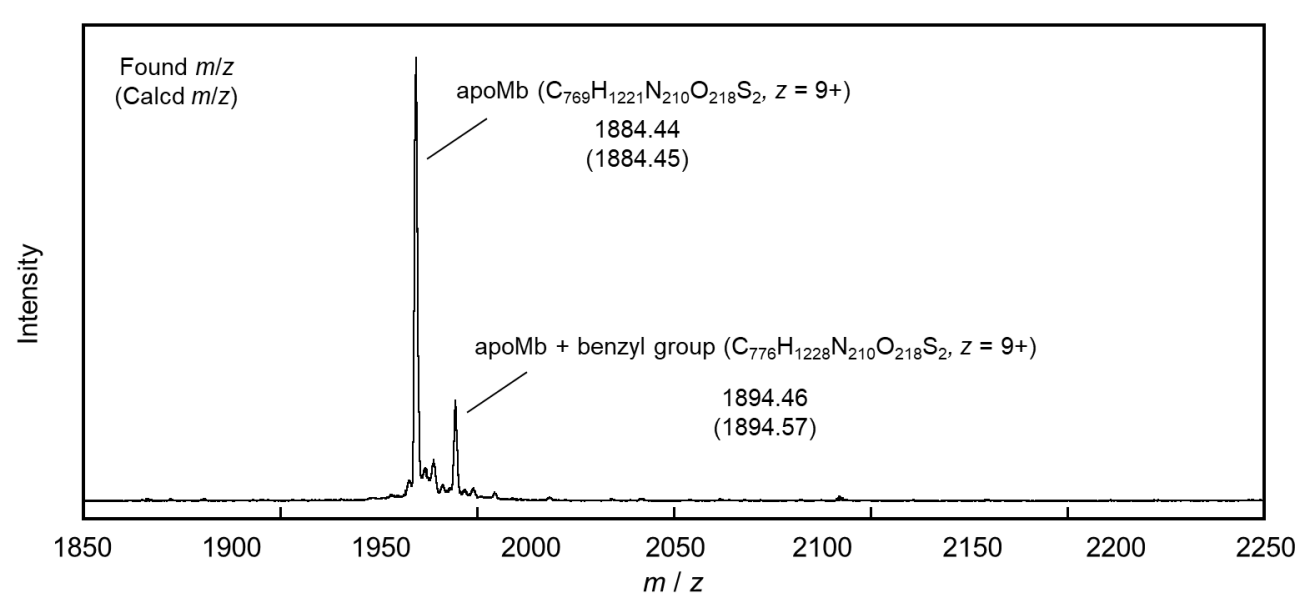

(b)

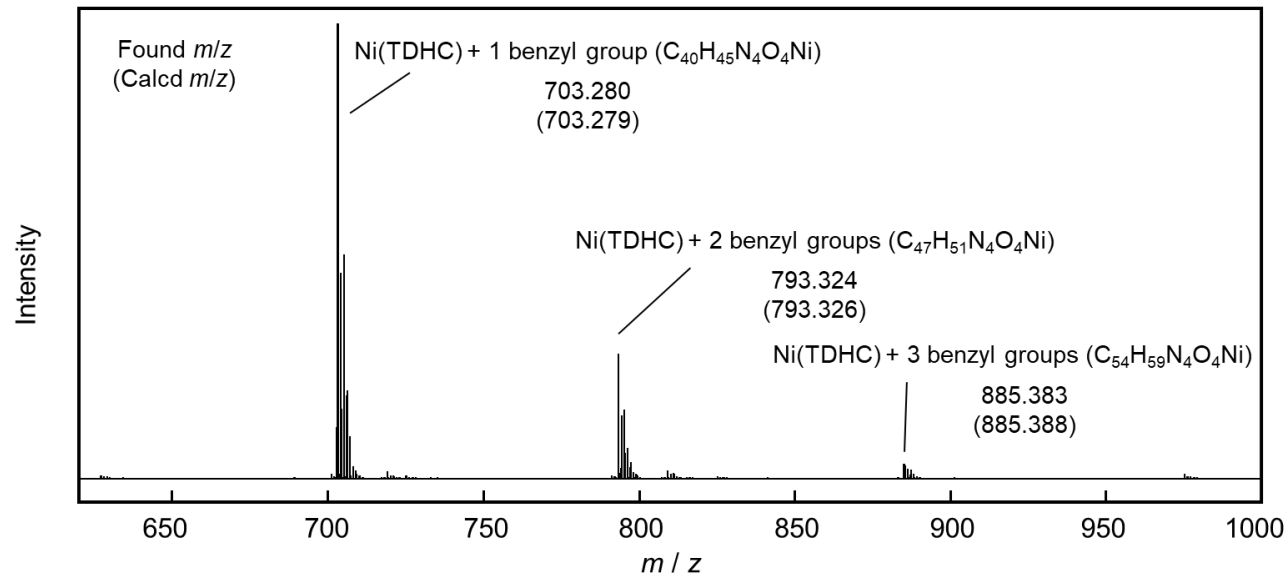

Figure S4. ESI-TOF mass spectra for (a) protein and (b) Ni(TDHC) complex of rMb(Ni(TDHC)) after the dehalogenation reaction with benzyl bromide. 
Table S4. Values of $\log k_{\mathrm{obs}}$ for reductive dehalogenation reaction of para-substituted benzyl bromides by $\left.\mathrm{rMb} \mathrm{Ni}(\mathrm{TDHC})\right)$ and tabulated Hammett substituent constants for para-substituents. ${ }^{\mathrm{a}}$

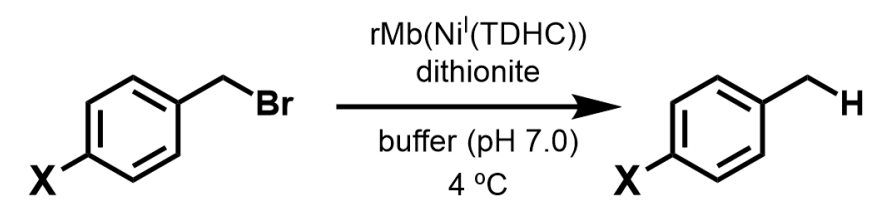

\begin{tabular}{|c|c|c|c|c|c|c|c|c|c|c|}
\hline substituent $(\mathbf{X})$ & $\log \left[k_{\mathrm{obs}}\left(\mu \mathrm{M} \cdot \mathrm{min}^{-1}\right)\right]$ & $\sigma_{\mathrm{P}}$ & $\sigma_{\mathrm{P}}^{+}$ & $\sigma_{\mathrm{P}}{ }^{-}$ & $\sigma_{\mathrm{mb}}$ & $\sigma_{\mathrm{JJ}}{ }^{\circ}$ & $\sigma_{\mathrm{j}}^{*}$ & $\sigma_{\mathrm{C}}{ }^{\circ}$ & $\sigma_{\alpha}{ }^{\cdot}$ & $\sigma_{\mathrm{F}}^{*}$ \\
\hline$-\mathrm{CH}_{3}$ & -0.61 & -0.17 & -0.31 & -0.17 & -0.29 & 0.15 & 0.39 & 0.11 & 0.015 & -0.02 \\
\hline$-\mathrm{H}$ & -0.41 & 0 & 0 & 0 & 0 & 0 & 0 & 0 & 0 & 0 \\
\hline$-\mathrm{I}$ & -0.41 & 0.18 & 0.14 & 0.27 & & & & & & 0.16 \\
\hline$-\mathrm{Br}$ & -0.26 & 0.23 & 0.15 & 0.25 & 0.13 & 0.23 & 0.2 & 0.13 & & 0.17 \\
\hline$-\mathrm{Cl}$ & -0.32 & 0.23 & 0.11 & 0.19 & 0.11 & 0.22 & 0.18 & 0.12 & 0.017 & 0.08 \\
\hline$-\mathrm{CF}_{3}$ & -0.14 & 0.54 & 0.61 & 0.65 & 0.49 & -0.01 & & 0.08 & 0.001 & \\
\hline$-\mathrm{CN}$ & 0.02 & 0.66 & 0.66 & 1.0 & 0.86 & 0.42 & 0.41 & 0.46 & 0.043 & 0.34 \\
\hline$-\mathrm{NO}_{2}$ & 0.29 & 0.78 & 0.79 & 1.27 & 0.86 & 0.36 & 0.76 & & & 0.27 \\
\hline
\end{tabular}

${ }^{\mathrm{a}}$ Data were collected from following sources, $\sigma_{\mathrm{P}},{ }^{\mathrm{S} 6} \sigma_{\mathrm{P}}{ }^{+},{ }^{\mathrm{S} 6} \sigma_{\mathrm{P}}{ }^{-\mathrm{S} 6} \sigma_{\mathrm{mb}},{ }^{\mathrm{S} 7} \sigma_{\mathrm{JJ}}{ }^{\mathrm{S} 8} \sigma_{\mathrm{J}}{ }^{\mathrm{S} 9} \sigma_{\mathrm{C}}{ }^{\circ}{ }^{\mathrm{S} 10} \sigma_{\alpha} \cdot{ }^{\circ}{ }^{\mathrm{S} 11}$ and $\sigma_{\mathrm{F}} \cdot{ }^{\circ \mathrm{S} 12}$ 

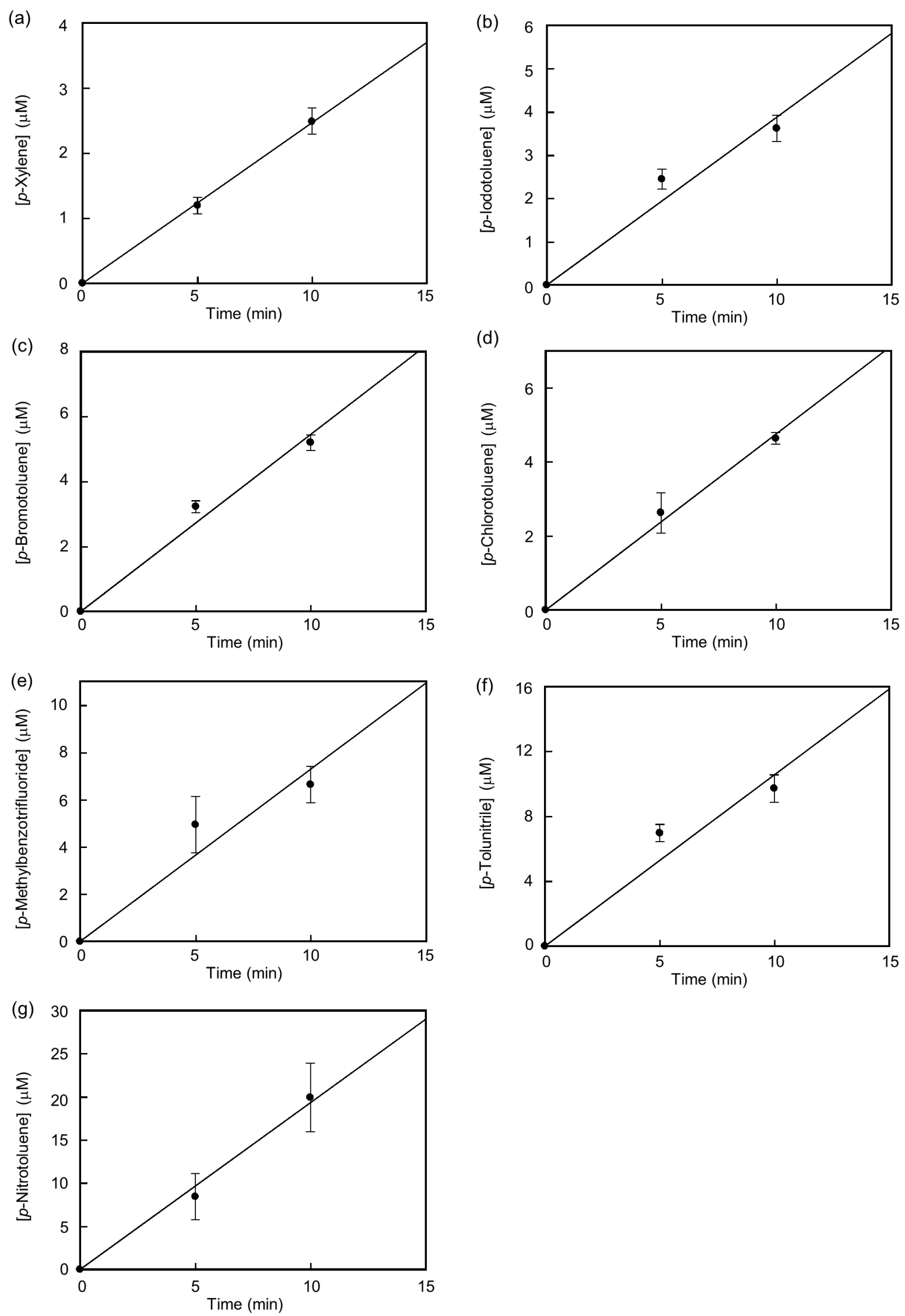

Figure S5. Time-course plots of para-substituted toluene derivatives generation by the reductive dehalogenation of para-susbstituted benzyl bromides ((a) - $\mathrm{CH}_{3}$, (b) - I, (c) - Br, (d) -Cl, (e) - $\mathrm{CF}_{3}$, (f) $\mathrm{CN}$, and $\left.(\mathrm{g})-\mathrm{NO}_{2}\right)$ using $\mathrm{rMb}\left(\mathrm{Ni}^{\mathrm{I}}(\mathrm{TDHC})\right)$. Conditions: $\left[\mathrm{rMb}\left(\mathrm{Ni}^{\mathrm{I}}(\mathrm{TDHC})\right)\right]=45 \mu \mathrm{M}$, [dithionite $]=$ 
$1.0 \mathrm{mM}$, [para-susbstituted benzyl bromide $]=1.0 \mathrm{mM}$ in potassium phosphate buffer $(100 \mathrm{mM}, \mathrm{pH}$ 7.0) containing $1 \%(v / v)$ acetonitrile at $4{ }^{\circ} \mathrm{C}$ under an $\mathrm{N}_{2}$ atmosphere.

(a)

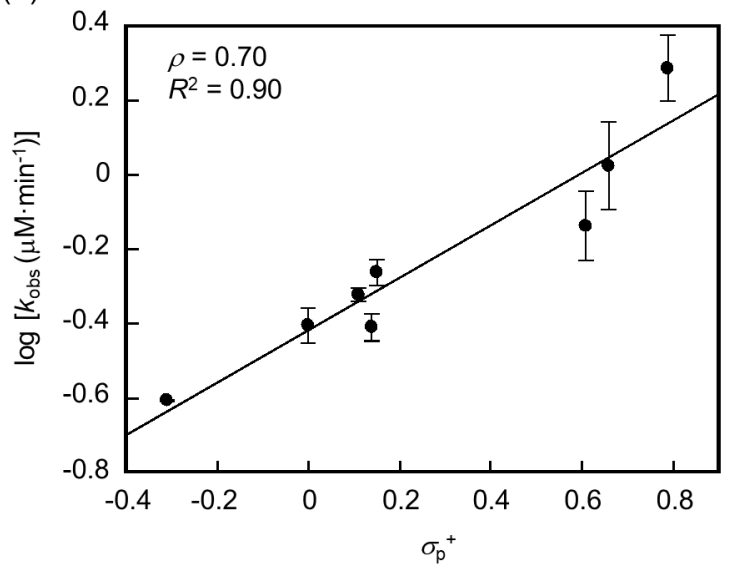

(b)

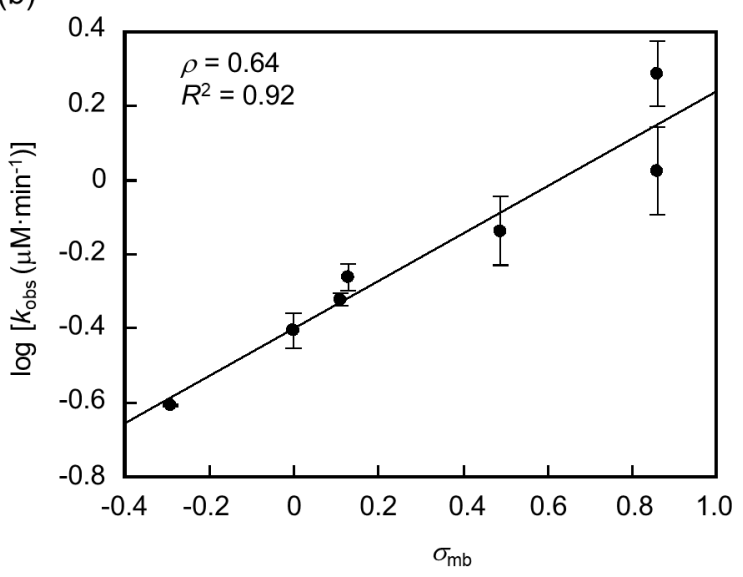

Figure S6. Plots of $\log k_{\mathrm{obs}} \mathrm{vs} \sigma_{\mathrm{P}}{ }^{+}$(a) and $\sigma_{\mathrm{mb}}(\mathrm{b})$ for the reductive dehalogenation reaction of parasubstituted benzyl bromides by $\mathrm{rMb}\left(\mathrm{Ni}^{\mathrm{I}}(\mathrm{TDHC})\right)$. 
(a)

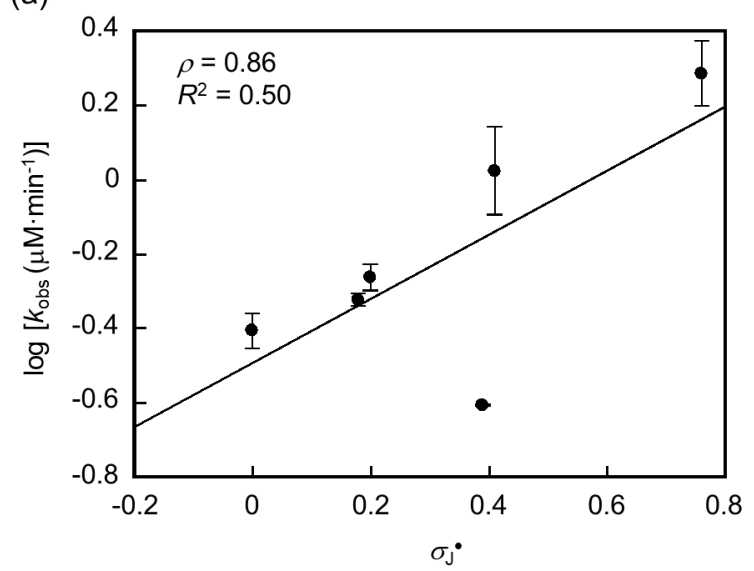

(c)

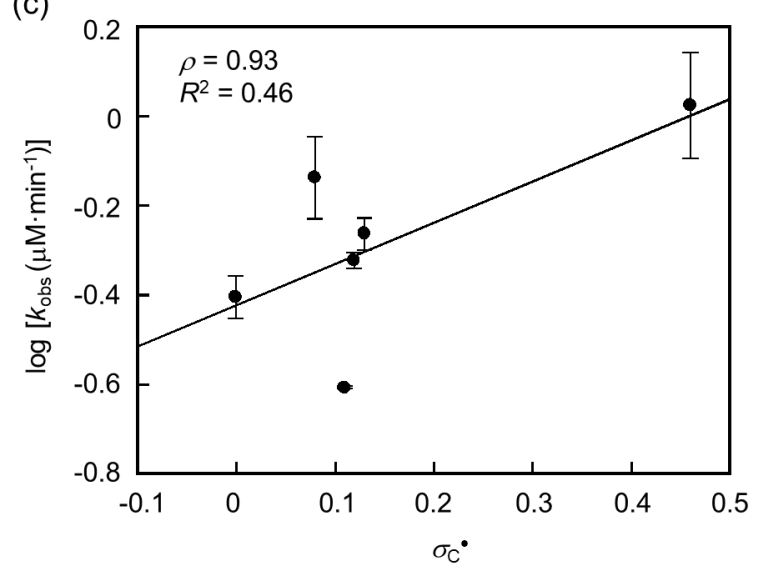

(e)

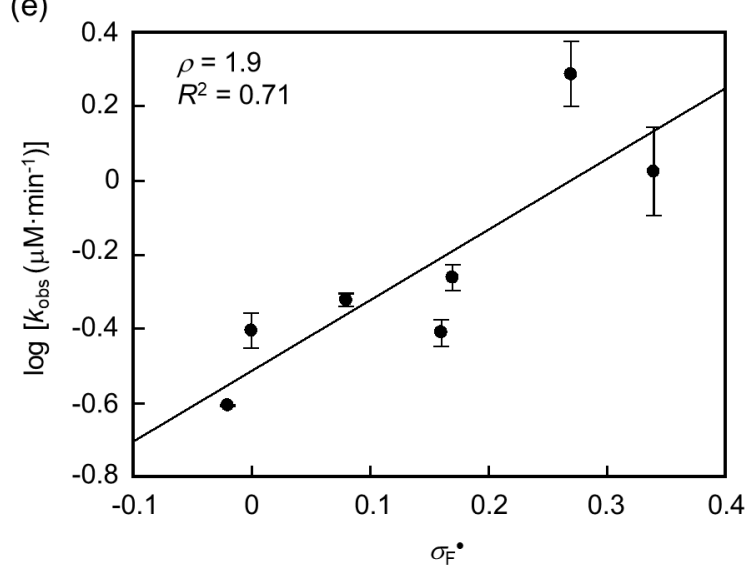

(b)

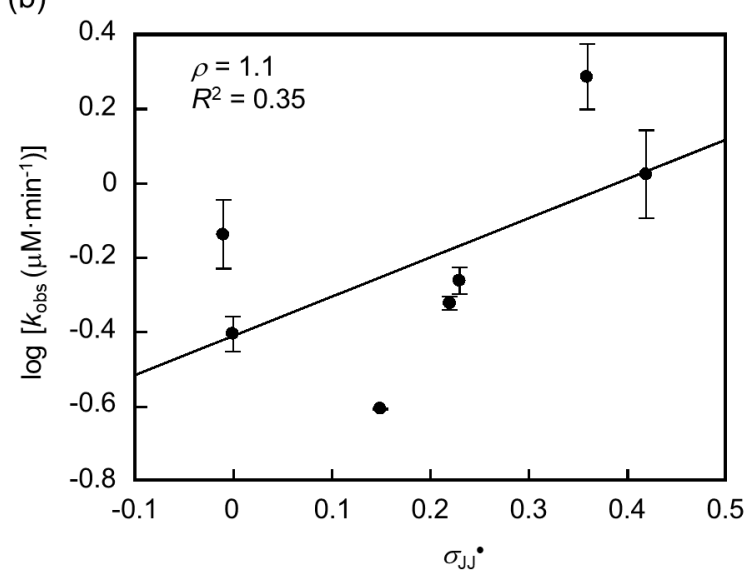

(d)

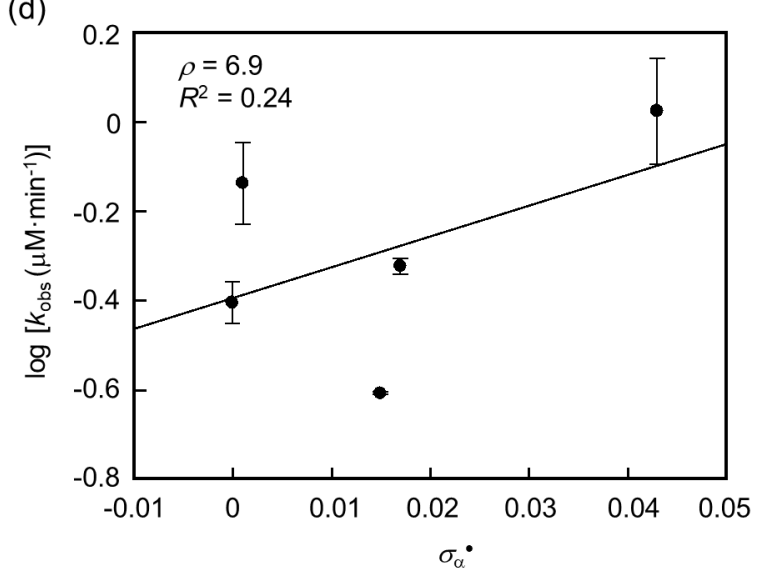

Figure S7. Plots of $\log k_{\mathrm{obs}}$ vs (a) $\sigma_{\mathrm{J}}$, (b) $\sigma_{\mathrm{JJ}}$, (c) $\sigma_{\mathrm{C}}$; (d) $\sigma_{\alpha}$, and (e) $\sigma_{\mathrm{F}}{ }^{\circ}$ for the reductive dehalogenation reaction of para-substituted benzyl bromides by $\mathrm{rMb}\left(\mathrm{Ni}^{\mathrm{I}}(\mathrm{TDHC})\right)$. 
(a)

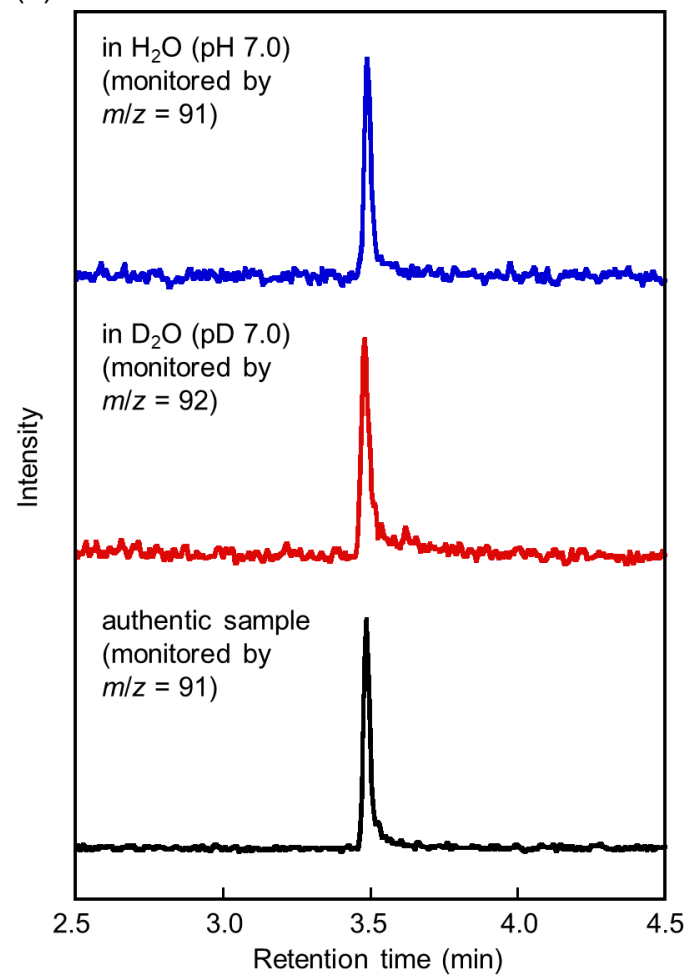

(b)
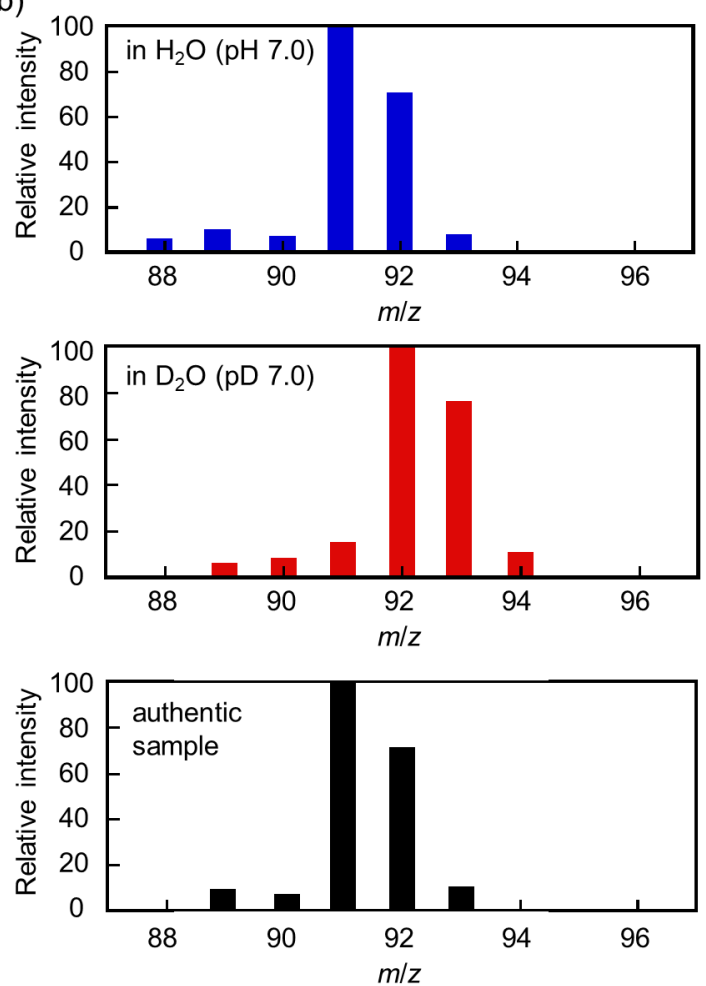

Figure S8. (a) GC/MS traces of the reaction using benzyl bromide in phosphate buffer ( $\mathrm{pH} 7.0$ in $\mathrm{H}_{2} \mathrm{O}$ ) monitoring $m / z=91$ (top), in $\mathrm{D}_{2} \mathrm{O}$ (pD 7.0 in phosphate buffer) monitoring $m / z=92$ (middle), and authentic toluene monitoring $m / z=91$ (bottom). (b) Mass spectra of toluene $\left(\mathrm{C}_{6} \mathrm{H}_{5} \mathrm{CH}_{3}\right)$ generated in $\mathrm{H}_{2} \mathrm{O}\left(\mathrm{pH} 7.0\right.$ in phosphate buffer) (top) and toluene- $d_{1}\left(\mathrm{C}_{6} \mathrm{H}_{5} \mathrm{CH}_{2} \mathrm{D}\right)$ generated in $\mathrm{D}_{2} \mathrm{O}(\mathrm{pD} 7.0$ in phosphate buffer) (middle) and of authentic toluene $\left(\mathrm{C}_{6} \mathrm{H}_{5} \mathrm{CH}_{3}\right)$ (bottom). Conditions: $\left[\mathrm{rMb}\left(\mathrm{Ni}^{\mathrm{I}}(\mathrm{TDHC})\right)\right]=45 \mu \mathrm{M},[$ dithionite $]=1.0 \mathrm{mM}$, [benzyl bromide $]=1.0 \mathrm{mM}$ in $100 \mathrm{mM}$ potassium phosphate buffer $\left(\mathrm{pH} 7.0\right.$ in $\left.\mathrm{H}_{2} \mathrm{O}\right)$ or $\left(\mathrm{pD} 7.0\right.$ in $\left.\mathrm{D}_{2} \mathrm{O}\right)$ containing $1 \%(v / v)$ acetonitrile for $2 \mathrm{~h}$ at $4{ }^{\circ} \mathrm{C}$ under an $\mathrm{N}_{2}$ atmosphere. 
(a)

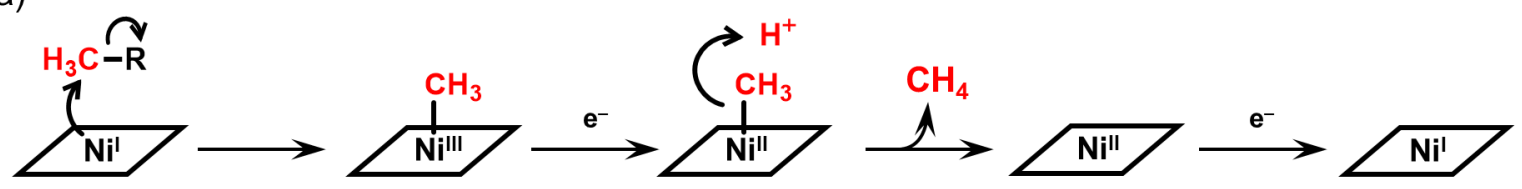

(b)

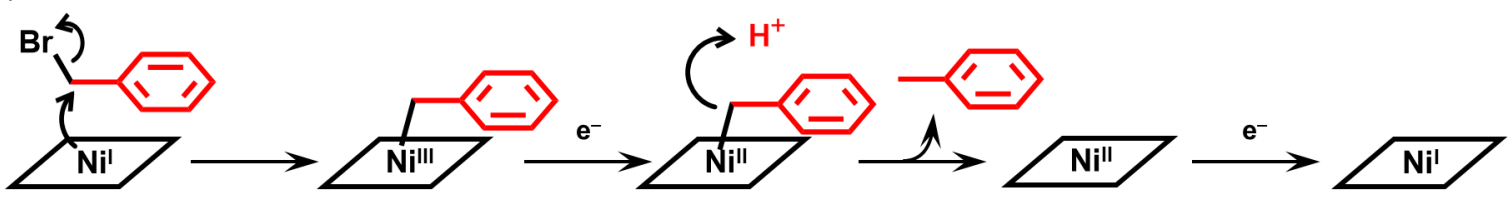

(c)

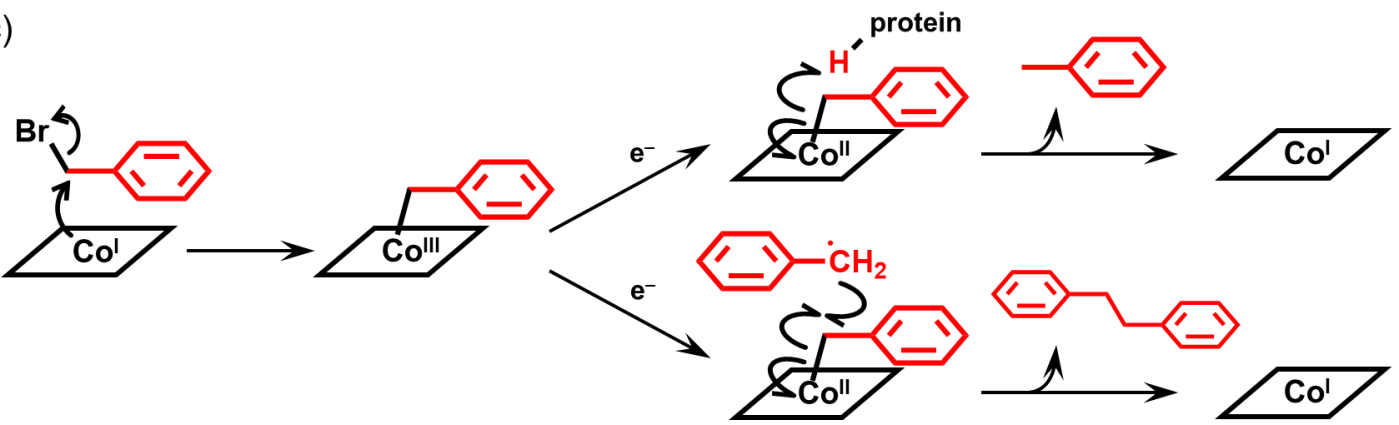

Figure S9. Suggested reaction mechanisms for (a) methane generation from methyl donor using $\mathrm{rMb}\left(\mathrm{Ni}^{\mathrm{I}}(\mathrm{TDHC})\right)$, (b) reductively debromination reaction of benzyl bromide using $\mathrm{rMb}\left(\mathrm{Ni} \mathrm{T}^{\mathrm{I}}(\mathrm{TDHC})\right.$ ), and (c) debromination reaction of benzyl bromide using $\mathrm{rMb}\left(\mathrm{Co}^{\mathrm{I}}(\mathrm{TDHC})\right)$. These mechanisms are proposed for the productive pathway, and actual reaction in the present system includes nonproductive pathway toward inactivation of the cofactor to yield the benzyl adduct at the meso position. 


\section{References}

[S1] Oohora, K.; Miyazaki, Y.; Hayashi, T. Myoglobin Reconstituted with Ni

Tetradehydrocorrin as a Methane-Generating Model of Methyl-coenzyme M Reductase. Angew. Chem. Int. Ed. 2019, 58, 13813-13817.

[S2] Hayashi, T.; Morita, Y.; Mizohata, E.; Oohora, K.; Ohbayashi, J.; Inoue, T.; Hisaeda, Y. $\mathrm{Co}(\mathrm{II}) / \mathrm{Co}(\mathrm{I})$ reduction-induced axial histidine-flipping in myoglobin reconstituted with a cobalt tetradehydrocorrin as a methionine synthase model. Chem. Commun. 2014, 50, 12560-12563.

[S3] Teale, F. W. J.; Cleavage of the haem-protein link by acid methylethylketone Biochim. Biophys. Acta 1959, 35, 543.

[S4] Scheller, S.; Goenrich, M.; Mayr, S.; Thauer, R. K.; Jaun, B. Intermediates in the Catalytic Cycle of Methyl Coenzyme M Reductase: Isotope Exchange is Consistent with Formation of a $\sigma$ Alkane-Nickel Complex. Angew. Chem. Int. Ed. 2010, 49, 8112-8115.

[S5] Ajvazi, N.; Stavber, S. Direct halogenation of alcohols with halosilanes under catalyst- and organic solvent-free reaction conditions. Tetrahedron Lett. 2016, 57, 2430-2433.

[S6] Hansch, C.; Leo, A.; Taft, R. W. A Survey of Hammett Substituent Constants and Resonance and Field Parameters. Chem. Rev. 1991, 91, 165-195.

[S7] Ji, G.-Z.; Jiang, X.-K.; Zhang, Y.-H.; Yuan, S.-G.; Yu, C.-X.; Shi, Y.-Q.; Zhang, X.-L.; Shi, W.-T. The spin delocalization substitutent parameter $\sigma^{\circ}$ J. 5 . Correlation analysis of ${ }^{19} \mathrm{~F}$ chemical shifts of substituted trifluorostyrenes. The unresolved polar substituent parameter $\sigma_{\mathrm{mb}}$. J. Phys. Org. Chem. 1990, 3, 643-650.

[S8] Jiang, X.-K.; Ji, G.-Z. A Self-Consistent and Cross-Checked Scale of Spin-Delocalization Substituent Constants, the $\sigma^{\circ}$ JJ Scale. J. Org. Chem. 1992, 57, 6051-6056.

[S9] Ağirbaş, H.; Jackson, R. A. Free Radical Reactions in Solution. Part 9. Further $\sigma^{*}$ Values from Decomposition of Substituted Dibenzylmercurials. J. Chem. Soc., Perkin Trans. 2 1983, 739-742.

[S10] (a) Creary, X.; Mehrsheikh-Mohammadi, M. E.; McDonald, S. Methylenecyclopropane Rearrangement as a Probe for Free Radical Substituent Effects. $\sigma^{*}$ Values for Commonly Encountered Conjugating and Organometallic Groups. J. Org. Chem. 1987, 52, 3254-3263. (b) Creary, X. Super Radical Stabilizers. Acc. Chem. Res. 2006, 39, 761-771.

[S11] Dust, J. M.; Arnold, D. R. Substituent Effects on Benzyl Radical ESR Hyperfine Coupling Constants. The $\sigma_{\alpha} \cdot$ Scale Based upon Spin Delocalization. J. Am. Chem. Soc. 1983, 105, 1221-1227.

[S12] Fisher, T. H.; Meierhoefer, A. W. Substituent Effects in Free-Radical Reactions. A Study of 4-Substituted 3-Cyanobenzyl Free Radicals. J. Org. Chem. 1978, 43, 224-228. 